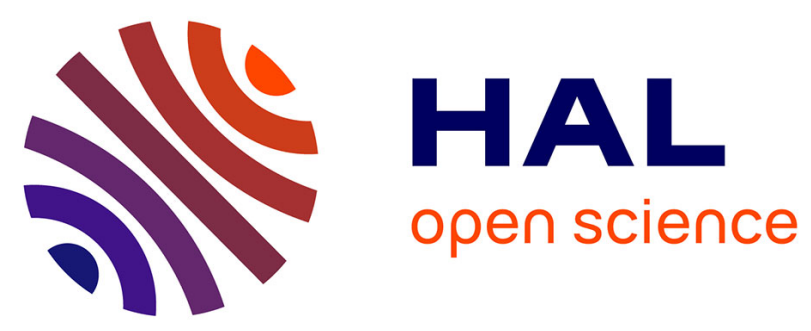

\title{
Galileo E1 OS/SoL acquisition, tracking and data demodulation performances for Civil Aviation
}

Olivier Julien, Christophe Macabiau, Jean-Luc Issler, Lionel Ries

\section{To cite this version:}

Olivier Julien, Christophe Macabiau, Jean-Luc Issler, Lionel Ries. Galileo E1 OS/SoL acquisition, tracking and data demodulation performances for Civil Aviation. NAVITEC 2010, 5th ESA Workshop on Satellite Navigation Technologies and European Workshop on GNSS Signals, Dec 2010, Noordwijk, Netherlands. pp 1-8, 10.1109/NAVITEC.2010.5708081 . hal-01022204

\section{HAL Id: hal-01022204 \\ https://hal-enac.archives-ouvertes.fr/hal-01022204}

Submitted on 29 Sep 2014

HAL is a multi-disciplinary open access archive for the deposit and dissemination of scientific research documents, whether they are published or not. The documents may come from teaching and research institutions in France or abroad, or from public or private research centers.
L'archive ouverte pluridisciplinaire HAL, est destinée au dépôt et à la diffusion de documents scientifiques de niveau recherche, publiés ou non, émanant des établissements d'enseignement et de recherche français ou étrangers, des laboratoires publics ou privés. 


\section{Galileo E1 OS/SoL Acquisition, Tracking and Data Demodulation Performances for Civil Aviation}

\author{
Olivier Julien, Christophe Macabiau \\ Laboratoire de Traitement du Signal et des \\ Télécommunications \\ Ecole Nationale de l'Aviation Civile (ENAC) \\ Toulouse France
}

\author{
Jean-Luc Issler, Lionel Ries \\ Centre National d'Etude Spatiales (CNES) \\ Toulouse, France
}

\begin{abstract}
This article presents a methodology related to the computation of civil aviation acquisition, tracking and data demodulation thresholds adapted to the case of Galileo E1 OS/SoL signal. These thresholds are compared against worst case link budgets representing operational scenarios. It is shown that these thresholds provide a positive margin although these theoretical results would probably require a deeper analysis and confirmation through extensive testing.
\end{abstract}

\section{Keywords-component; Galileo, Civil Aviation, Thresholds}

\section{INTRODUCTION}

The use of Galileo by the civil aviation community requires a thorough analysis of the signals' performance to ensure that a Galileo receiver on-board can fulfill a given set of requirements in worst-case operational conditions. These requirements can be found in civil aviation standardization documents such as, for instance, the RTCA DO-229D for the Minimum Operational Performance Standards (MOPS) for the GPS L1 C/A / WAAS airborne equipment [1]. An equivalent document is currently being written by the EURopean Organization for Civil Aviation Equipment (EUROCAE) for the use of Galileo by the civil aviation community.

Certain requirements, such as the initial acquisition time, tracking accuracy, cycle slip occurrence rate or the Word Error Rate (WER), are directly linked with the receiver's acquisition, tracking and data demodulation functions. The performance of these three functions can be expressed as a function of the signal power to noise power spectral density ratio $(\mathrm{C} / \mathrm{N} 0)$. However, according to the structure of the considered signal, the receiver conditions (such as signal dynamics) or the receiver complexity, the performance of the different aforementioned functions might differ. Moreover, depending upon the system characteristics, the required minimal performance of some functions might change (for instance the WER).

Consequently, as part of the assessment of the Galileo E1 OS/SoL signal for civil aviation, it is necessary to compute the minimum $\mathrm{C} / \mathrm{N}_{0}$ values for which the acquisition, tracking and data demodulation functions will fulfill the civil aviation requirements in operational scenarios, considering standard techniques and an airborne Galileo receiver with a reasonable complexity. However, certain requirements not being yet fully defined for Galileo, it is also interesting to provide performance assessment as a function of the received $\mathrm{C} / \mathrm{N}_{0}$ so that it will be easy to set the $\mathrm{C} / \mathrm{N}_{0}$ threshold once all the requirements are fully defined. The computation of these thresholds (or performance assessment) for the Galileo E1 OS/SoL signal will constitute the main goal of the paper.

The paper is organized as follows:

- section II introduces the Galileo E1 OS/SoL signal and correlator output model used through the paper,

- section III provides the computation of the acquisition threshold,

- section IV provides the computation of the phase tracking threshold,

- section $\mathrm{V}$ provides the computation of the data demodulation threshold, and

- section VI concludes on the findings of the paper.

\section{SignAL MODEL}

\section{A. Galileo E1 SoL Received Signal Model}

The incoming Galileo E1 SoL signal can be modelled at the Intermediate Frequency (IF) as [2]:

$s(t)=A\left(\begin{array}{c}c_{D}(t-\tau) d_{D}(t-\tau) C B O C_{-}(t-\tau) \\ -c_{P}(t-\tau) s_{C}(t-\tau) C B O C_{+}(t-\tau)\end{array}\right)$

with

$$
\begin{aligned}
& \text { CBOC }_{-}(t)=\sqrt{10 / 11} \text { BOC }_{1}(t)-\sqrt{1 / 11} \text { BOC }_{6}(t) \\
& \text { CBOC }_{+}(t)=\sqrt{10 / 11} \text { BOC }_{1}(t)+\sqrt{1 / 11} \text { BOC }_{6}(t) \\
& \text { BOC }_{x}(t)=\operatorname{sign}\left(\sin \left(x \cdot 2 \pi \cdot 1.023 e^{6} \cdot t\right)\right)
\end{aligned}
$$

where

- $A$ is the amplitude of the incoming signal at the correlator input,

- $c_{P}$ and $c_{D}$ are the spreading sequences carried by the pilot and data components. They have a chipping rate equal to 1.023 Mega-chips per second (Mcps) and a length of 4092 chips. 
- $d_{D}$ represents the symbols of the I/NAV navigation message [2] modulating the data component. It has a rate of 250 symbols per second (sps).

- $s_{c}$ represents the secondary code present on the pilot component that has a length of 25 chips and a rate of $250 \mathrm{~Hz}$.

- $\tau$ is the delay of the spreading sequence,

- $f_{I F}$ is the intermediate frequency, and

- $\theta$ is the phase offset of the carrier.

\section{B. Receiver Type and Correlator Output Model}

In the following, the receiver will be assumed to be a narrowband $\mathrm{BOC}(1,1)$ receiver. The main reason for this choice is the desire to start this analysis considering only a very simple receiver. Indeed, if the simplest receiver is able to fulfill the civil aviation requirements, then more advanced receivers should also be able to fulfill these same requirements. The considered receiver architecture implies that:

- the equivalent front-end filter of the receiver takes only the first lobes of the Galileo E1 OS/SoL Power Spectral Density (PSD), which is $4 \mathrm{MHz}$ double-sided.

- the receiver generates locally only a BOC $(1,1)$ local replica to process the data and pilot components of the Galileo E1 OS/SoL signal

With this in mind, and assuming that the data and pilot component spreading sequences are quasi-orthogonal, the correlator output model can be approximated by $[3,4]$ :

$$
\begin{aligned}
I_{D}(k) & =\sqrt{\frac{P}{4}} d_{D}(k) \tilde{R}_{D, L}\left(\varepsilon_{\tau}\right) \operatorname{sinc}\left(\pi \varepsilon_{f} T_{I}\right) \cos \left(\varepsilon_{\varphi}\right)+n_{I_{L}}(k) \\
Q_{D}(k) & =\sqrt{\frac{P}{4}} d_{D}(k) \tilde{R}_{D, L}\left(\varepsilon_{\tau}\right) \operatorname{sinc}\left(\pi \varepsilon_{f} T_{I}\right) \sin \left(\varepsilon_{\varphi}\right)+n_{Q_{L}}(k) \\
I_{P}(k) & =\sqrt{\frac{P}{4}} S_{c}(k) \tilde{R}_{P, L}\left(\varepsilon_{\tau}\right) \operatorname{sinc}\left(\pi \varepsilon_{f} T_{I}\right) \cos \left(\varepsilon_{\varphi}\right)+n_{I_{\mathrm{L}}}(k) \\
Q_{P}(k) & =\sqrt{\frac{P}{4}} s_{c}(k) \tilde{R}_{P, L}\left(\varepsilon_{\tau}\right) \operatorname{sinc}\left(\pi \varepsilon_{f} T_{I}\right) \sin \left(\varepsilon_{\varphi}\right)+n_{Q_{\mathrm{L}}}(k)
\end{aligned}
$$

with

$\tilde{R}_{X, Y}(x)=\int_{-\infty}^{+\infty} H(f) F_{X}(f) F_{Y}(f) e^{-2 j \pi f x} d f$.

where

- $P$ represents the power of the incoming signal at the correlator input (and thus includes a certain number of losses due to the front-end),

- the subscripts $D$ and $P$ are for the incoming "data component" and "pilot component" respectively,

- the subscripts $L$ is for the local spreading sequence, a BOC $(1,1)$ local replica in the present case,

- $h$ and $H$ are the equivalent front-end filter impulse response and transfer function respectively,

- $F_{X}$ is the Fourier transform of the signal $X$,

- $\tilde{R}_{X, Y}$ is the correlation function between the incoming spreading sequence $X$ and the locally generated spreading sequence $Y$ taking into account the effect of the front-end filter $H$,
- $\varepsilon_{\tau}$ is the code delay error between the incoming code delay and the one estimated by the receiver,

- $\varepsilon_{\varphi}$ is the phase error (in the middle of the correlation interval) between the incoming carrier and the locally generated carrier,

- $\varepsilon_{f}$ is the frequency error between the incoming carrier and the locally generated carrier,

- $T_{I}$ is the correlation time,

- $n_{I_{D}}, n_{Q_{D}}$ are the noise components of the two discriminator outputs associated with the data component respectively; $n_{I_{P}}$, and $n_{Q_{P}}$ are the noise components of the 2 discriminator outputs associated with the pilot component respectively. All these noise components are all uncorrelated, and:

$$
\operatorname{Var}\left[n_{I_{D}}\right]=\operatorname{Var}\left[n_{Q_{D}}\right]=\operatorname{Var}\left[n_{I_{P}}\right]=\operatorname{Var}\left[n_{Q_{P}}\right]=\frac{N_{0}}{4 T_{I}} \beta
$$

- $N_{0} / 2$ is the equivalent double-sided thermal noise PSD at the correlator input. In the context of this study, it also includes the interference components that can be equivalently represented by a white noise component at the correlator input.

- $\beta=\int_{-\infty}^{+\infty}|H(f)|^{2}\left|F_{L}(f)\right|^{2} d f$ is the loss of noise power due to the front-end filter. Note that since the data and pilot components are processed with a BOC $(1,1)$ receiver, $\beta$ is the same for their associated correlator output.

The correlation functions $\tilde{R}_{P, L}$ and $\tilde{R}_{D, L}$ are represented in Figure 1 using the receiver settings described earlier. It can be seen that filtering the higher frequency components and processing the signal using a $\mathrm{BOC}(1,1)$ receiver creates very similar data and pilot correlation functions.

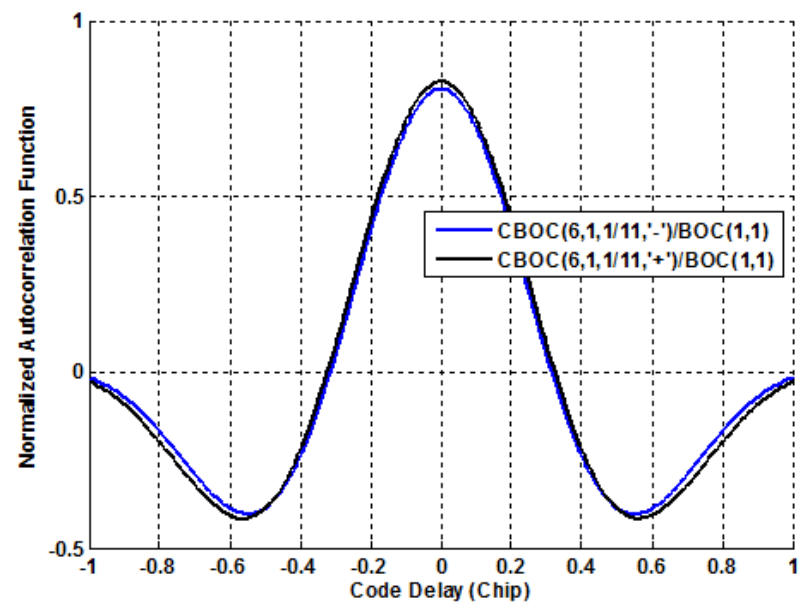

Figure 1.Galileo E1 SoL Data and Pilot Correlation Functions Using a BOC $(1,1)$ Receiver with a 4-MHz (Double-Sided) Front-End Filter

\section{ACQUISITION THRESHOLD}

\section{A. Acquisition Requirement}

The current version of the Galileo Minimum Operational Performance Standard (MOPS) for the use of Galileo by civil 
aviation has the following requirement on the initial acquisition time:

The acquisition time from application of power to the first valid position fix for either E5a, E5b or E1 shall be less than 5 minutes with a 95\% probability under the following conditions:

The receiver

- shall be initialised to a latitude and longitude position within 60 nautical miles

- shall be initialised to within one minute of UTC time.

- shall be velocity aided within $30 \mathrm{~m} / \mathrm{s}$

- shall have valid almanac data and unobstructed satellite visibility

- will be subjected to the interference conditions of Appendix $B$

In this context "valid" means all of the following conditions are met:

- The determined position meets the accuracy / integrity requirements for at least one minute following the first position fix.

The analysis presented in this section aims at providing minimum $\mathrm{C} / \mathrm{N}_{0}$ values required at the correlator output level for a given minimalist receiver architecture so that the initial acquisition requirement can be fulfilled.

\section{B. Methodology and Assumptions}

The methodology applied here reproduces the methodology used by the RTCA to assess the acquisition threshold of the GPS L1 C/A and the GPS L5 [1; 3]. This methodology has however been adapted to the case of Galileo.

It is well-known that a minimum of 4 Galileo satellites have to be acquired in order to compute a first position. Once these 4 satellites have been acquired, it is then necessary to check the integrity of the computed position. Based on this, it seems fair to split the 5 min allocated to the initial acquisition into $[1 ; 3]$ :

- $2.5 \mathrm{~min}$ for the acquisition of the first 4 satellites, and

- $2.5 \mathrm{~min}$ for the remaining tasks:

overification,

odata demodulation,

oAchievement of accuracy and integrity requirement (settling of tracking loops and demodulation of, in some instances, multiple copies of messages, etc...).

The acquisition of the first 4 satellites is here assumed to be sequential. It can thus be assumed that there is a specific acquisition time allocated to each satellite: $T_{i}$ sec for the acquisition of the $\mathrm{i}^{\text {th }}$ satellite. Moreover, it is assumed that the receiver acquisition strategy is to search for the satellite with the highest elevation angle (since the receiver is supposed to have access to the almanac, this is a likely strategy). Finally, the receiver is assumed to search once every bin of the acquisition matrix and thus to spend exactly $T_{i}$ sec to search for the $\mathrm{i}^{\text {th }}$ satellite. This is clearly a worst case scenario that allows a simplification of the methodology.

The acquisition of the first satellite is clearly the most difficult one since there is the largest unknown in the Doppler value. [3] assessed the initial Doppler uncertainty to be driven by the receiver oscillator at turn-on (TCXO with a 1 part per million stability), leading to a Doppler uncertainty of $+/-1500 \mathrm{~Hz}$. However, once that first satellite has been acquired, this significantly reduces the Doppler uncertainty since it is then possible to estimate the bias between the expected Doppler computed using the almanac and the actual acquired Doppler. This leads to a remaining Doppler uncertainty of $+/-150 \mathrm{~Hz}$. It is then easier to acquire the $2^{\text {nd }}-4^{\text {th }}$ satellites. To take that into account, and to simplify the methodology, only 2 acquisition time allocations will be considered:

- the acquisition time of the $1^{\text {st }}$ satellite: $T_{1}$, and

- the acquisition time of each of the $2^{\text {nd }}-4^{\text {th }}$ satellite: $T_{2-4}$.

Taking into account (a) the satellite selection strategy, (b) the receiver acquisition methodology described earlier, and (c) assuming that the acquisition of each satellite is independent, the probability of acquisition $P_{\text {Acq }}$ within 2.5 min of the first 4 satellites can be decomposed into:

$P_{A c q}=P_{A c q, 1} \cdot P_{A c q, 2} \cdot P_{A c q, 3} \cdot P_{A c q, 4}$

where $P_{A c q, n}$ can be decomposed using conditional probability:

$P_{\text {Acq, } n}=\int_{-\infty}^{\infty+} P_{d, n}(x) \cdot P\left(\left(C / N_{0}\right)_{n}=x\right) d x$

with :

- $P_{d, n}(x)$ is the probability of acquiring a satellite with a $\mathrm{C} / \mathrm{N}_{0}$ equal to $x$ within $T_{n}$ sec. using the aforementioned receiver strategy,

- $P\left(\left(C / N_{0}\right)_{n}=x\right)$ is the probability that the $\mathrm{C} / \mathrm{N}_{0}$ of the $\mathrm{n}$-th satellite is equal to $x$.

It is clear that the acquisition of the fourth satellite will be more difficult since this satellite should also be the lowest and thus with a lower $\mathrm{C} / \mathrm{N}_{0}$. As a consequence, (3) can be upper bounded by:

$P_{A c q}>P_{A c q, 1} \cdot\left(P_{A c q, 4}\right)^{4}$

Considering the worst case scenario where $\left(C / N_{0}\right)_{\min , n}$ represents the lowest elevation possible of the $n$-th satellite, (4) can be lower bounded by:

$P_{\text {Acq }}>\left(\begin{array}{c}P_{d, n}\left(C / N_{0}=\left(C / N_{0}\right)_{\min , 1}\right) \\ \cdot\left(P_{d, n}\left(C / N_{0}=\left(C / N_{0}\right)_{\min , n}\right)\right)^{3}\end{array}\right)$ 
Initial simulations considering a 27-satellite constellation with a maximum of 2 coincident satellite failures showed that the minimum elevation of the highest Galileo satellite is around $48^{\circ}$, while the minimum elevation of the fourth highest satellite is around $18^{\circ}$.

Regarding the initial acquisition time requirement, and assuming that the verification phase will always be successful, it is then necessary to have $P_{A c q} \geq 0.95$. To ensure this, it is required to target probability values in (5) that fulfill $P_{A c q} \geq 0.95$. For instance, it would be sufficient to show that considering the aforementioned receiver acquisition strategy:

$\left\{\begin{array}{l}P_{d, n}\left(C / N_{0}<\left(C / N_{0}\right)_{\min , 1}\right)=0.98 \\ P_{d, n}\left(C / N_{N_{0}}<\left(C / N_{0}\right)_{\min , n}\right)=0.99\end{array}\right.$

The idea is then to make sure that these probabilities can be fulfilled with the considered receiver. If this is the case, the threshold could then be chosen below $\min \left\{\left(C / N_{0}\right)_{\min , 1},\left(C / N_{0}\right)_{\min , n}\right\}$ to ensure that there is an operational margin.

\section{Computation of the Probability of Detection}

The acquisition architecture is based on the non-coherent use of the data and pilot components of the Galileo E1 OS/SoL signal, similarly to [3].

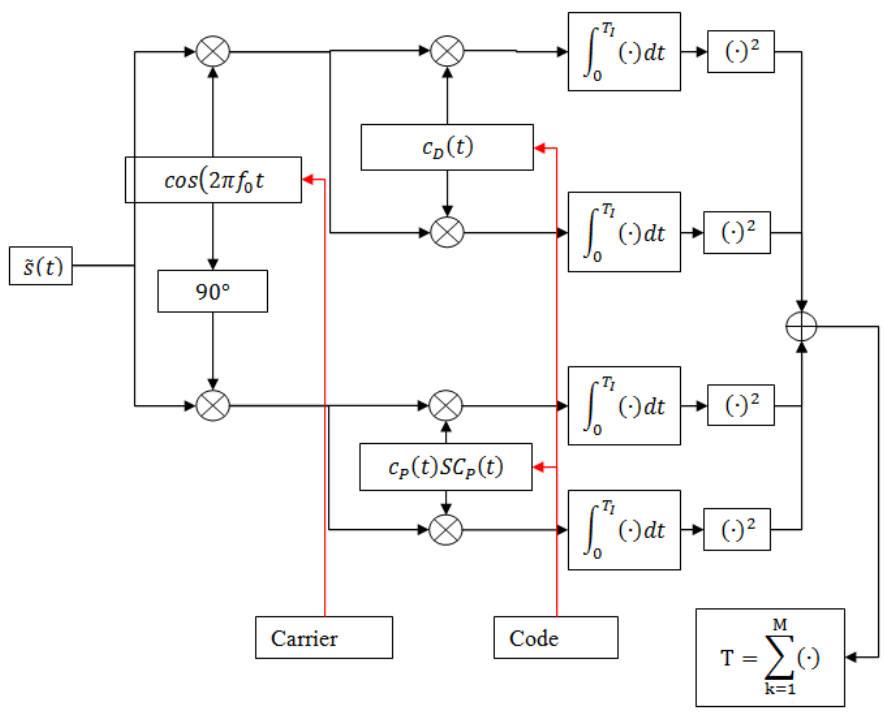

Figure 2.Galileo E1 OS/SoL Receiver Acquisition Structure

The considered acquisition detector is then:

$T=\sum_{k=1}^{M}\left(I_{D}^{2}(k)+Q_{D}^{2}(k)+I_{P}^{2}(k)+Q_{P}^{2}(k)\right)$

where $M$ is referred to as the number of non-coherent summations.
The computation of the probability of detection is done through the well known Neyman-Pearson detector where 2 hypothesis are considered: H0: the desired signal is not present, and H1: the desired signal is present.

Let us first assume that the useful signal is absent, but that there is a strong interfering signal coming from another Galileo satellite that creates a high cross-correlation peak. Under this hypothesis, the acquisition detector normalized by the correlator output noise variance $\left(\bar{T}_{0}\right)$ will have a non-central Chi-square distribution with $4 \mathrm{M}$ degrees of freedom and a non-centrality parameter equal to:

$\bar{\lambda}_{0}=\frac{P_{J} T_{I}}{\beta N_{0}} \sum_{k=1}^{M}\left(\tilde{R}_{D_{J}, L}^{2}\left(\varepsilon_{\tau_{\mathrm{j}}}\right)+\tilde{R}_{P_{J}, L}^{2}\left(\varepsilon_{\tau_{\mathrm{j}}}\right)\right)$

where

- $P_{J}$ represents the power of the interfering signal at the correlator input (and thus includes a certain number of losses due to quantization, insertion losses, etc...)

- $\varepsilon_{\tau_{\mathrm{j}}}$ is the code delay between the local replica and the incoming interferer that generates the worst case cross-correlation peak.

The variation of the code delay at the E1 frequency can be considered close to constant within a few hundred milliseconds. Thus, the non-centrality parameter can be approximated by:

$\bar{\lambda}_{0}=M \frac{P_{J} T_{I}}{\beta N_{0}}\left(\tilde{R}_{D_{J}, L}^{2}\left(\varepsilon_{\tau_{\mathrm{j}}}\right)+\tilde{R}_{P_{J}, L}^{2}\left(\varepsilon_{\tau_{\mathrm{j}}}\right)\right)$

Knowing the distribution of the random variable $\bar{T}_{0}$, it is then easy to find the threshold $T h$ such that $P\left(\bar{T}_{0}>T h\right)=$ $P\left(T_{0}>T h \cdot \frac{N_{0}}{4 T_{I}} \beta\right)=P_{f a}$ for a desired probability of false alarm $P_{f a}$.

Let us now assume that the useful signal is present. In this case, the acquisition detector normalized by the correlator output noise variance $\left(\bar{T}_{1}\right)$ will have a non-central Chisquare distribution with $4 \mathrm{M}$ degrees of freedom and a noncentrality parameter equal to:

$\bar{\lambda}_{1}=2 T_{I} \cdot \frac{P}{N_{0}} \cdot L_{\text {Corr }} \cdot \sum_{k=1}^{M} \operatorname{sinc}^{2}\left(\pi \varepsilon_{f} T_{I}\right) L_{I n c}\left(\varepsilon_{\tau}\right)$

Assume that the incoming signal characteristics do not change during the non-coherent summation. Then:

$\bar{\lambda}_{1}=2 M T_{I} L_{\text {Corr }, A c q} \cdot \frac{P}{N_{0}} \cdot L_{I n c}\left(\varepsilon_{\tau}\right) \cdot \operatorname{sinc}^{2}\left(\pi \varepsilon_{f} T_{I}\right)$

where

- $L_{I n c}=\frac{\tilde{R}_{D, \mathrm{~L}}^{2}\left(\varepsilon_{\tau}\right)+\tilde{R}_{P, \mathrm{~L}}^{2}\left(\varepsilon_{\tau}\right)}{\tilde{R}_{D, \mathrm{~L}}^{2}(0)+\tilde{R}_{P, \mathrm{~L}}^{2}(0)}$ represents the incremental losses (shown in Figure 3 ) which means the losses due to the code delay error, and

- $L_{\text {Corr }, A c q}=\frac{\tilde{R}_{D, \mathrm{~L}}^{2}(0)+\tilde{R}_{P, \mathrm{~L}}^{2}(0)}{\beta}$ represents the correlation losses specific to the acquisition technique, which means the $\mathrm{C} / \mathrm{N}_{0}$ losses due to the correlation process. 


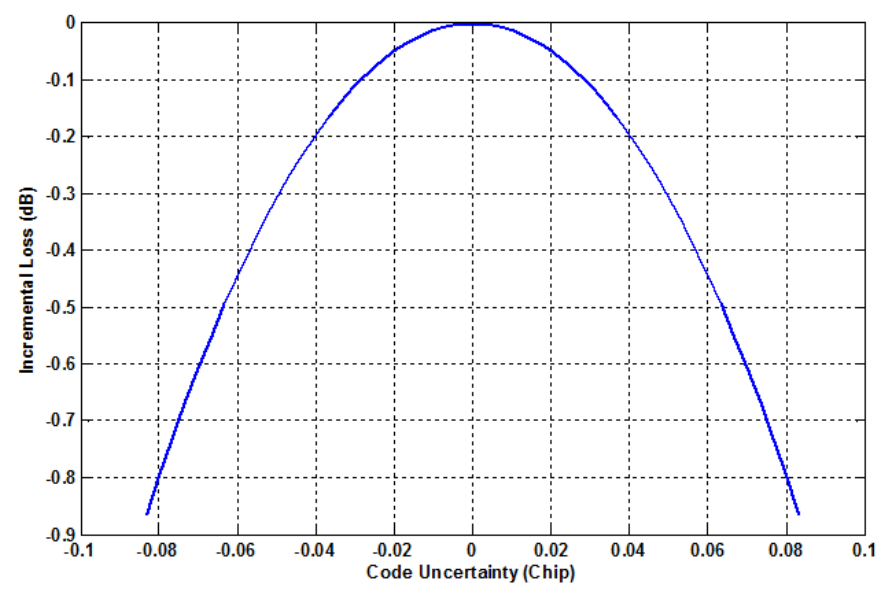

Figure 3.Galileo E1 Incremental Losses Using a BOC $(1,1)$ Receiver with a 4$\mathrm{MHz}$ (Double-Sided) Front-End Filter

As it was discussed in section II.C, the threshold should represent the $\mathrm{C} / \mathrm{N}_{0}$ at the correlator output, thus, in (10), $L_{C o r r, A c q} \cdot \frac{P}{N_{0}}$ will be replaced by $\frac{C}{N_{0}}$. Taking this into account, (10) becomes:

$\bar{\lambda}_{1}=2 M T_{I} \frac{C}{N_{0}} \cdot L_{I n c}\left(\varepsilon_{\tau}\right) \cdot \operatorname{sinc}^{2}\left(\pi \varepsilon_{f} T_{I}\right)$

It can be seen that the non-central parameter will depend upon a number of factors:

- The post-correlation $C / N_{0}$,

- $\quad$ The coherent integration time $T_{I}$,

- The number of non-coherent summations $M$,

- The front-end filter through $L_{I n c}$, and

- The misalignments $\varepsilon_{\tau}$ and $\varepsilon_{f}$.

Knowing the distribution of the random variable $\bar{T}_{1}$, it is then easy to find the probability of detection $P_{d}$ since $P\left(\bar{T}_{1}>T h\right)=P\left(T_{1}>T h \cdot \frac{N_{0}}{4 T_{I}} \beta_{D}\right)=P_{d}$. It is thus possible to find the $C / N_{0}$ value that will make the initial acquisition requirement fulfilled according to the presented methodology.

\section{Computation of the Acquisition Threshold}

As already explained, the considered receiver acquisition strategy will search once every bin of the uncertainty space. This means that the number of non-coherent summations equals:

$M_{i, \max }=\left\lfloor\frac{N T_{i}}{\left.2 \cdot\left[\frac{\Delta c}{\delta c}\right] \cdot \mid \frac{\Delta D}{\delta D}\right] \cdot T_{I}}\right\rfloor$

where

- $[\cdot]$ and $[\cdot]$ represent the rounding to the lower and upper integer values respectively.

- The code delay and Doppler uncertainty are referred to as $\Delta c$ and $\Delta D$.
- The acquisition uses code delay and Doppler bins of width $\delta c$ and $\delta D$

- The receiver has $N$ complex correlators that can be used to search simultaneous $N / 2$ bins (2 complex correlators per bin).

Once $M_{i, \max }$ has been computed, it is then possible to compute the probability of detection of the satellite as a function of the incoming $\mathrm{C} / \mathrm{N}_{0}$ and the number of complex correlators based on the results exposed in section III.C.

To take into account the incremental losses, [3] proposes to compute the mean probability of detection considering that the code delay and Doppler errors are uniformly distributed within the correct search bin:

$\bar{P}_{d}=E\left[P_{d}\left(\varepsilon_{\tau} ; \varepsilon_{f}\right)\right]_{\varepsilon_{\tau} \in\left[-\frac{\delta c}{2} ; \frac{\delta c}{2}\right] ; \varepsilon_{f} \in\left[-\frac{\delta D}{2} ; \frac{\delta D}{2}\right]}$

The idea is then to select, as the acquisition threshold, the minimum $\mathrm{C} / \mathrm{N}_{0}$ (for a given number of complex correlators) that is such that:

- the probability of detection is greater than 0.98 for the 1st satellite and 0.99 for the remaining satellites(as already expressed in (6)), and

- it is lower than $\left(C / N_{0}\right)_{m i n, i}$ for a reasonable number of correlators. For this, it is required to compute the link budget.

\section{E. Acquisition Scenario Link Budget}

The link budget presented here is very similar to the one computed in [3] with the exception that the inter- and intraGNSS interference has not been thoroughly computed, but only conservatively approximated. It is here assumed that the aircraft is en-route and located around the QZSS hot-spot (where the inter-system interference is the highest). It takes into account:

- the minimum received power from the Galileo E1 OS satellite equals $-157.25 \mathrm{dBW}$

- the implementation losses comprising the quantization noise and correlation losses. In the case of the narrow-band $\mathrm{BOC}(1,1)$ receiver, the acquisition correlation losses equal $1.1 \mathrm{~dB}$ (assuming a rectangular equivalent front-end filter). Besides, a 1-bit quantization brings an extra $1.6 \mathrm{~dB}$. Consequently, the implementation losses were taken equal to $2.7 \mathrm{dBs}$.

- the minimum antenna gains. The antenna gain model is shown in TABLE 1 , it shows that the gain at $48^{\circ}$ elevation is $-0.6 \mathrm{~dB}$, while the gain at $18^{\circ}$ elevation is $-1.6 \mathrm{~dB}$.

- The presence of interference, which impact on the correlator output is the same as that of an equivalent white noise with a given $N_{0, \text { interf }}$ at the entrance of the correlator input. The considered interference [3], given the scenario, are:

- on-board installed avionics emissions,

- Aeronautical Mobile Satcom Unwanted RFI. 
- Non-aeronautical PED aggregate

- Pulse RFI with $1 \%$ duty cycle

- The presence of inter- and intra-system interference (GPS, Galileo, COMPASS and the different SBAS)

The equivalent $N_{0, \text { interf }}$ for all these interference is pessimistically estimated to be $-195.9 \mathrm{dBW} / \mathrm{Hz}$.

TABle 1 - Galileo E1 AnTenna Gain Model

\begin{tabular}{|c|c|c|}
\hline $\begin{array}{c}\text { Elevation } \\
\text { Angle }\end{array}$ & Min Gain (dBic) & Max. Gain (dBic) \\
\hline$\left[\mathbf{7 5}^{\circ} ; \mathbf{9 0}^{\circ}\right]$ & +0.5 & +3 \\
\hline$\left[\mathbf{2 0}^{\circ} ; \mathbf{7 5}^{\circ}\right]$ & $\begin{array}{c}-1+0.0231 \times\left(\mathrm{elev}^{\circ}-\right. \\
\left.20^{\circ}\right)\end{array}$ & $\begin{array}{c}+2.5+0.0077 \times\left(\mathrm{elev}^{\circ}-\right. \\
\left.10^{\circ}\right)\end{array}$ \\
\hline$\left[\mathbf{1 0}^{\circ} ; \mathbf{2 0}^{\circ}\right]$ & $-7+0.3 \times \mathrm{elev}^{\circ}$ & $\begin{array}{c}+2.5+0.0077 \times\left(\mathrm{elev}^{\circ}-\right. \\
\left.10^{\circ}\right)\end{array}$ \\
\hline$\left[\mathbf{0}^{\circ} ; \mathbf{1 0}^{\circ}\right]$ & $-7+0.3 \times \mathrm{elev}^{\circ}$ & $-2+0.45 \times \mathrm{elev}^{\circ}$ \\
\hline
\end{tabular}

This rough budget link provides minimum worst case $\mathrm{C} / \mathrm{N}_{0}$ (at the correlator output) values of 35.4 and $34.4 \mathrm{~dB}-\mathrm{Hz}$ for a satellite at an elevation of $48^{\circ}$ and $18^{\circ}$ respectively.

\section{F. Results and Conclusions}

There are a certain number of assumptions to take to realize the computation of the acquisition threshold:

- The aircraft will be assumed to have stored the navigation message and to have undergone a short power outage that required a warm start acquisition.

- $T_{I}=4 m s$. This is due to the fact that the coherent correlation time is limited to the duration of the navigation symbol duration on the data component and the secondary code chip duration on the pilot component.

- $T_{1}=105 \mathrm{~s}$ and $T_{2-4}=15 \mathrm{~s}$. These values were chosen to represent the fact that it is more difficult to acquire the first satellite due to the higher uncertainty on the Doppler.

- $\delta c=1 / 6$ chips. The usual bin size chip for the GPS C/A is $1 / 2$. Since the correlation function of the $\operatorname{BOC}(1,1)$ has a slope approximately 3 times higher than that of the BPSK(1), the bin size was reduced by 3 .

- $\delta D=125$ chips. The Doppler bin size is half of $1 / T_{I}$ (typically).

- $P_{f a}=2.5 e^{-4}$.

- The interfering Galileo satellite has an estimated $\mathrm{C} / \mathrm{N}_{0}$ at the correlator output equal to $47.5 \mathrm{~dB}-\mathrm{Hz}$.

Based on these parameters, the $C / N_{0}$ values for which $P_{d, 1}\left(C / N_{0}\right)=0.98$ and $P_{d, 4}\left(C / N_{0}<\left(C / N_{0}\right)_{\text {min, } n}\right)=0.99$ are shown in Table 2. From these results, it can be seen that a reasonable acquisition threshold could be around $33.7 \mathrm{~dB}-\mathrm{Hz}$ for both. Indeed, in this case, the number of complex correlators required is only around 300 (still significantly higher than the number of correlators typically used in a GPS $\mathrm{C} / \mathrm{A}$ receiver), however, this still seems acceptable.
TABLE 2 - ACQUiSITION THRESHOLDS AS A FunCTION OF THE NUMBER OF COMPLEX CORRELATORS

\begin{tabular}{|c|c|c|}
\hline \multirow{2}{*}{$\begin{array}{l}\text { Number of } \\
\text { Complex } \\
\text { Correlators }\end{array}$} & \multicolumn{2}{|c|}{ BOC Receiver } \\
\hline & $\begin{array}{l}\text { Req. C/ } \mathrm{N}_{0, \text { eff }} \text { for } \\
1^{\text {st }} \text { sat. (dB-Hz) }\end{array}$ & $\begin{array}{c}\text { Req. } \mathrm{C} / \mathrm{N}_{0, \text { eff }} \text { for } \\
2^{\text {nd_-4th sat. }} \\
\text { (dB-Hz) }\end{array}$ \\
\hline 100 & 36.8 & 37.1 \\
\hline 200 & 34.8 & 34.5 \\
\hline 300 & 33.7 & 33.6 \\
\hline 400 & 33.0 & 32.8 \\
\hline $\begin{array}{l}\text { Worst Case } \\
\text { Link Budget }\end{array}$ & 35.4 & 34.4 \\
\hline
\end{tabular}

\section{TRACKING THRESHOLD}

Since code tracking is significantly more robust than phase tracking, this section will only focus on phase tracking thresholds. In general, the most constraining performance criteria for phase tracking is the cycle slip occurrence rate $[1,3]$. Although the requirement regarding the cycle slip occurrence rate is not yet clear for Galileo E1 OS/SoL, the requirement used for GPS L5 in [3] was aligned on the requirement used for WAAS in [1], which is $10^{-5}$ cycle slip/sec. This value will thus be considered here as an example.

The aircraft will be assumed en-route over the QZSS hot spot enduring the maximum normal manoeuvre dynamics of 0.25 $\mathrm{g} / \mathrm{s}$.

Phase tracking is assumed to take place only on the pilot component using a Q-discrimiator $[5,6]$. The PLL is assumed to be of the 3rd order, to have an equivalent loop bandwidth $B_{L}$ equal to $10 \mathrm{~Hz}$, and to be implemented according to [7].

\section{A. Sources of Errors Affecting the PLL}

This section only reminds the different sources of phase tracking errors and the associated values applying to the present paper $[5,8,9]$.

\section{Thermal Noise}

The impact of thermal noise on phase tracking can be modeled through a white Gaussian carrier tracking phase jitter with a variance equal to:

$\sigma_{\varepsilon_{\mathrm{Th}}}^{2}=\frac{2 B_{L}}{\frac{C}{N_{0}}}\left(\mathrm{rad}^{2}\right)$

Note that in the present case, the $\mathrm{C} / \mathrm{N}_{0}$ represents the correlator output $\mathrm{C} / \mathrm{N}_{0}$ associated to the whole signal (data+pilot).

\section{Oscillator Phase Noise}

[2] specifies that the phase noise spectral density of the unmodulated carrier will allow a second-order PLL with 10 
$\mathrm{Hz}$ one-sided noise bandwidth to track the carrier to an accuracy of 0.04 radians RMS. In the present case, this same value will be used, although a3rd order PLL is considered.

The receiver is assumed to use a stable TCXO. The induced tracking jitter standard deviation for the specified PLL is $0.0873 \mathrm{rad}\left(5^{\circ}\right)$.

[9] provides a model in order to compute the phase tracking jitter due to the vibration of the oscillator. Following this model, the resulting phase tracking jitter standard deviation is $0.0593 \mathrm{rad}$.

As a consequence, the total oscillator phase noise jitter will be taken equal to $\sigma_{\varepsilon_{O s c}}=0.1129 \mathrm{rad}$

\section{Dynamics}

The effect of a constant jerk on a 3rd order PLL will be a tracking bias $\gamma$. This bias can be computed according to [7]. For our specific case, the maximum jerk during normal manoeuvres equals $0.25 \mathrm{~g} / \mathrm{s}$. This provides a bias $\gamma=$ $0.1713 \mathrm{rad}$.

No multipath is considered here since the aircraft is assumed en-route.

\section{B. Cycle Slip Occurrence Rate}

The main figure of merit that will be looked at is the cycle slip occurrence rate. The probability to have a cycle slip within one second can be modeled for a 1st order PLL using [8]:

$$
P=1-e^{-\frac{1}{T}}
$$

with

$\bar{T}=\frac{\pi}{2 B_{L} \gamma} \tanh \left(\frac{\pi \gamma}{\sigma_{\varepsilon_{\varphi}}^{2}}\right)\left[J_{0}^{2}\left(\frac{1}{\sigma_{\varepsilon_{\varphi}}^{2}}\right)+2 \sum_{n=1}^{+\infty}(-1)^{n} \frac{J_{n}^{2}\left(\frac{1}{\sigma_{\varepsilon_{\varphi}}^{2}}\right)}{1+\left(\frac{n \sigma_{\varepsilon_{\varphi}}^{2}}{\gamma}\right)^{2}}\right]$

where

- $\sigma_{\varepsilon_{\varphi}}^{2}=\sigma_{\varepsilon_{\mathrm{Th}}}^{2}+\sigma_{\varepsilon_{O S c}}^{2}$, and

- $J_{i}$ is the $\mathrm{n}$-th order modified Bessel function of the 1 st kind

For a 3rd order PLL, the rule of thumb is to use the previous formula adding an equivalent $2 \mathrm{~dB}$ to the actual tested $\mathrm{C} / \mathrm{N}_{0}$. The results are shown in Figure 4. It can be seen that the usual target of $10^{-5}$ cycle slip per second is reached for a total (data+pilot) post-correlation $\mathrm{C} / \mathrm{N}_{0}$ around $25 \mathrm{~dB}-\mathrm{Hz}$, which is significantly below the minimum received $\mathrm{C} / \mathrm{N}_{0}$ according to the link budget.

\section{Link Budget}

The associated link budget is the same as the one realized for the acquisition case, but with the difference that the worst case is now to consider the satellite that is at the lowest usable elevation, which is specified to be $10^{\circ}$. At this elevation, the antenna gain is $-4 \mathrm{~dB}$. Consequently, the minimum received $\mathrm{C} / \mathrm{N}_{0}$ should be around $32 \mathrm{~dB}-\mathrm{Hz}$. Therefore, there is a comfortable margin between the cycle slip occurrence rate threshold and the worst case link budget.

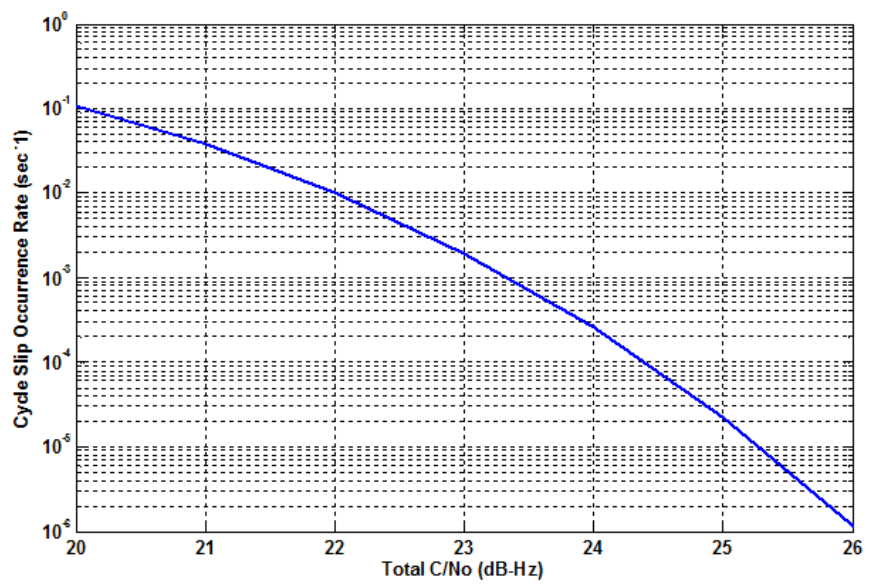

Figure 4.Cycle Slip Occurrence Rate Using a BOC $(1,1)$ Receiver with a 4$\mathrm{MHz}$ (Double-Sided) Front-End Filter

\section{DATA DEMODULATION THRESHOLD}

The requirement regarding phase data demodulation threshold is still not clear regarding the Galileo E1 signal due to the different structure of the message carried by Galileo E1 OS/SoL signal (referred to as I/NAV) compared to the GPS signals. As a reference, the WER taken for GPS L1 C/A and WAAS is $10^{-3}$ [3].

The same scenario as for the section IV will be assumed.

The structure of the Galileo I/NAV message is detailed in [2]. The useful message has a bit rate of $125 \mathrm{~Hz}$ and is encoded using a convolutional coding of constraint 7 with a rate of $1 / 2$. Consequently, the transmitted symbol rate is $250 \mathrm{~Hz}$.

Considering that the symbols are binary, the Symbol Error Rate (SER) considering a phase tracking error $\varepsilon_{\varphi}$ is given by [8]:

$P_{S}\left(\varepsilon_{\varphi}\right)=\frac{1}{2} \operatorname{erfc}\left(\sqrt{\frac{E_{S}}{N_{0}}} \cos \left(\varepsilon_{\varphi}\right)\right)$

where $E_{S}=\frac{C}{2} T_{I}$ is the symbol energy in the case of Galileo since the data is only present on the data component.

Consequently, the mean SER for the Galileo receiver can be estimated using the traditional expectation computation:

$S E R=\int_{-\pi}^{\pi} P_{b}(x) f_{\varepsilon_{\varphi}}(x) d x$

where $f_{\varepsilon_{\varphi}}$ represents the probability density function of the phase tracking error $\varepsilon_{\varphi}$.

$f_{\varepsilon_{\varphi}}$ can be approximated by a Gaussian function:

$f_{\varepsilon_{\varphi}}(x)=\frac{1}{\sqrt{2 \pi} \sigma_{\varepsilon \varphi}} e^{-\frac{(x-\gamma)}{2 \sigma_{\varepsilon}^{2}}}$

Assuming soft decoding using a Viterbi decoder, the Bit Error Rate (BER) may then be tightly upper bounded using the following expression [8]:

$P_{b}\left(\varepsilon_{\varphi}\right)=\frac{1}{2}\left(36 D^{10}+211 D^{12}+1404 D^{14}+11633 D^{16}\right)$

with 
$D=e^{-\frac{1 E_{S}}{2 N_{0}} \cos ^{2}\left(\varepsilon_{\varphi}\right)}$

Consequently, the mean BER for the Galileo receiver can be estimated using:

$B E R=\int_{-\pi}^{\pi} P_{b}(x) f_{\varepsilon_{\varphi}}(x) d x$

Finally, it is difficult to really compute the Galileo E1 OS/SoL WER due to the structure of the I/NAV message. Indeed, the message structure is composed of pages composed of 2 subpages. Each subpage (referred to as odd and even) contain 120 coded (by the convolutional code) bits and 10 symbols (not coded by the convolutional code) and thus lasts for $1 \mathrm{sec}$. Finally, a CRC of length 24 bits is used that protects the first 202 bits across the odd and even pages. In this context we will consider a word as being 120- or 240-bit long. The resulting WER is:

$$
W E R=1-(1-B E R)^{N}
$$

where $N$ is the number of bits composing the word.

The results are shown in Figure 5 for the case of a worst case dynamics equal to $0.25 \mathrm{~g} / \mathrm{s}$ and the case without dynamics. It can be seen that, for instance, for a targeted WER of $10^{-3}$, the $\mathrm{C} / \mathrm{N}_{0}$ threshold is between 29.3 and $30.5 \mathrm{~dB}-\mathrm{Hz}$.

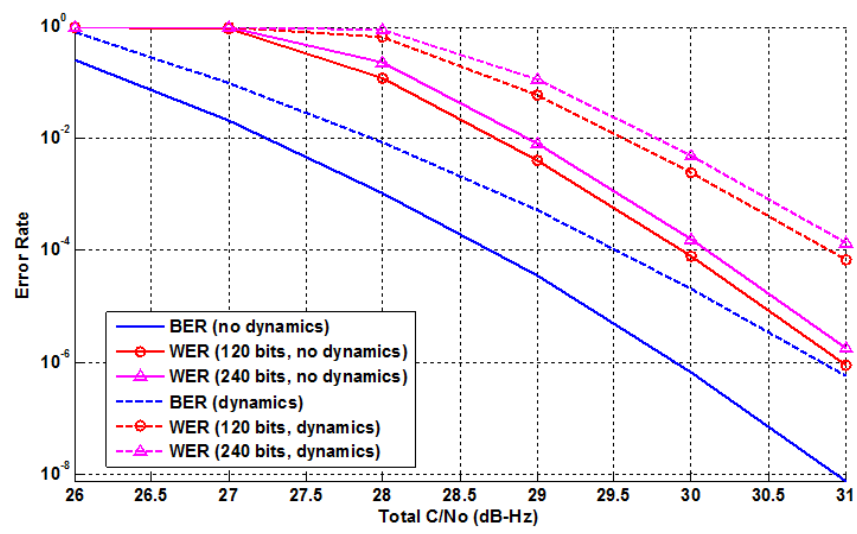

Figure 5 - BER and WER for the Galileo E1 OS/SoL Signal

The associated link budget is the same as the one realized for the phase tracking case. This means that there is a small positive margin left if a WER of $10^{-3}$ is targeted for data demodulation and that it is clearly necessary to better assess the worst-case inter- and intra- interference equivalent noise in the link to ensure that this does not create any problems.

In the specific case of the WER computation, it also seems necessary to realize extensive tests to verify the figures plotted in Figure 5. Indeed, it is noted in the literature [1,3] that it is very difficult to estimate the WER from the BER due to the occurrence of burst errors or due to the actual data structure.
Consequently, the results presented here have to be taken with great care and extensive testing is part of the next phase of this investigation.

\section{CONCLUSIONS AND FUTURE WORK}

This paper has presented the theoretical investigation of the acquisition, tracking and demodulation threshold for the use of the Galileo E1 SoL signal by the civil aviation community. Regarding the acquisition, a methodology directly derived from $[1,3]$ was presented. It showed that although the methodology is very pessimistic, it still allows for a fair margin with respect to the worst case link budget for acquisition. Regarding the cycle slip occurrence rate during tracking, it was also clear that there was a significant margin with respect to the link budget. Finally, there is still a slight uncertainty regarding the data demodulation threshold that calls for great care about the values presented. In particular extensive testing are needed to finalize this point.

\section{ACKNOWLEDGMENT}

The authors would like to thank Loic N'Dongollomo, student of the University of Toulouse for his contribution on this topic during his 5-month internship at ENAC

\section{REFERENCES}

[1] RTCA SC-159, Assessment of Radio Frequency Interference Relevant to the GNSS L1 Frequency Band, RTCA DO 235B, March 2008.

[2] European Commission (2010), European GNSS (Galileo) Open Service - Signal-In-Space Interface Control Document Issue 1, February.

[3] RTCA SC-159, Assessment of Radio Frequency Interference Relevant to the GNSS L5/E5a Frequency Band, RTCA DO 292, July 2004.

[4] Bastide, F., O. Julien, C. Macabiau, B. Roturier (2002), Analysis of L5/E5 Acquisition, Tracking and Data Demodulation Thresholds, Proceedings of the Institute of Navigation GPS (Portland, OR, USA, Sept. 24-27), pp. 2196-2207.

[5] Julien, O. (2005), Design of Galileo L1F Receiver Tracking Loops, Ph.D. thesis, Department of Geomatics Engineering, University of Calgary, UCGE Report 20227, available at the following address http://www.geomatics.ucalgary.ca/links/GradTheses.html.

[6] Hegarty, C. (1999), Evaluation of the Proposed Signal Structure for the New Civil GPS Signal at 1176.45 MHz, WN99W0000034, The MITRE Corporation

[7] Stephens S.A., and J. B. Thomas (1995), Controlled-Root Formulation for Digital Phase-Locked Loops, in IEEE Transactions on Aerospace and Electronic Systems, Vol. 31, No. 1, pp. 78-95.

[8] Hegarty, C., Analytical Derivation of the Maximum Tolerable In-Band Interference Levels for Aviation Applications of GNSS, Navigation: Journal of the Institute of Navigation, Vol. 44, No. 1, Spring 1997, pp. 25-34.

[9] Irsigler, M., and B. Eissfeller (2002), PLL Tracking Performance in Presence of Oscillator Phase Noise, GPS Solutions, Vol. 5, No. 4, pp. 45-54. 